\title{
Simulation-Based Education for the Rural Pediatric Trauma Team
}

\author{
Lilly Bayouth ${ }^{1 *}$, Shannon W. Longshore ${ }^{2}$ \\ 'Department of Surgery, Brody School of Medicine at East Carolina University, 600 Moye Blvd, Greenville, NC, USA \\ ${ }^{2}$ Division of Pediatric Surgery, Brody School of Medicine at East Carolina University, 600 Moye Blvd MA, 207, Greenville, NC, USA
}

Article Info

\section{Article Notes}

Received: June 25, 2018

Accepted: September 20, 2018

${ }^{*}$ Correspondence:

Dr. Lilly Bayouth MD, 600 Moye Blvd MA 207, Greenville, NC, 27834, USA; Telephone No: (252) 744-2832; Fax No: (252) 744-3457; Email: bayouthl14@ecu.edu.

(C) 2018 Bayouth L. This article is distributed under the terms of the Creative Commons Attribution 4.0 International License.

\section{Background}

Traumatic injury is the leading cause of death in children ages 0 - 17 years $^{1}$. Less than $10 \%$ of injured children receive care at a pediatric trauma center or children's hospital due to limited resources, distance, and a regional absence of pediatric surgeons and/or specialists ${ }^{2}$. Pediatric trauma patients are often taken to the nearest emergency department (ED), even that ED may not be equipped to provide the best care specific to the pediatric trauma patient's needs. For most of these EDs as well as prehospital transporters, pediatric trauma care, is a low-frequency, yet high-stakes, event that may generate significant anxiety among providers ${ }^{3}$. The limited resources and infrequent exposure makes it challenging for staff to maintain the skills necessary for efficient and successful care of the pediatric trauma patient. Pediatric Advanced Life Support (PALS) is an existing standardized resuscitation course but it offers minimal education in pediatric trauma care. Numerous studies have demonstrated the utility of in situ simulation-based training as both a tool for assessment and educational intervention with respect to trauma team function, efficacy, communication, and overall improvement in early trauma care provided, but few have evaluated its efficacy within the pediatric population ${ }^{4-7}$. The focus of this review is to provide the trauma community with an overview of the recent literature regarding the utility of simulation-based education initiatives within the field of pediatric trauma, encourage the application of these initiatives to rural or smaller community hospital settings, and discuss potential future research directions.

\section{Pediatric Emergency Care in the Rural Setting}

Despite advances in care and regionalization of trauma systems outcome disparities between urban and rural pediatric trauma patients persist $t^{8-9}$. Pediatric trauma care is a low frequency event. Not surprisingly, physicians serving rural communities report a greater need for continuing medical education with respect to pediatric emergency procedures as compared to those in urban centers ${ }^{10}$.

High quality trauma resuscitative care significantly improves mortality; however, staff may not have sufficient opportunities to maintain the skills necessary to provide this proficient care specific to the pediatric trauma patient. Pediatric trauma is a highly specialized field where patients require coordinated and timely execution of care during resuscitation. The lower volume of pediatric patients in non-pediatric facilities and rural settings, as well as the minimal opportunity for pediatric trauma education within the currently offered standardized resuscitative courses makes the 
implementation of team-training and pediatric trauma care concepts, as well as opportunity for review, crucial ${ }^{11-13}$

A prospective pilot study for a trauma outreach program utilizing in situ simulation-based education was recently initiated by a level 1 trauma center serving a largely rural population. They identified the three hospitals with highest volume of pediatric trauma referrals and completed in situ simulation-based training with video debriefing and surveys evaluating provider comfort level with pediatric trauma resuscitative tasks, pre and post training. Reassessment and focus group discussion comments revealed significant improvement in provider comfort level, successful resuscitation, proposed practice changes, and improved relationships and communication between the trauma center providers and their rural community hospital colleagues. One particular comment exemplifies the positive impact of simulation education on the relationship between the rural referring provider and the trauma center: "Putting a face to who we talk to really helps and is very beneficial. This helps build trust with the ER team and the trauma team." ${ }^{14}$.

\section{In Situ Pediatric Trauma Simulation-Based Education}

Simulation-based training is increasingly recognized as an education tool that can improve patient safety and medical practice as well as increase a provider's clinical knowledge base, procedural skills set, and communication with his/her healthcare team ${ }^{15-17}$. With respect to the trauma team, team function has been compared pre and post in situ simulation training ${ }^{4-7}$. Amiel et al, found significant improvement in teamwork and communication as well as other areas of treatment. Several studies targeting improvement in pediatric trauma room care using in situ trauma simulations with debriefing, have demonstrated positive results with overall improvements observed in teamwork, communication, resuscitative skills and/or patient care ${ }^{18-23}$.

Additional utility of simulation-based training lies in its ability to identify targets for performance improvement and assist in the correction of these performance deficiencies. A study conducted within 35 EDs used simulation training to evaluate the resuscitative care provided in injured pediatric patients. Deficiencies were present among all participating EDs, suggesting simulation-based education to be a valuable tool to help improve provider and team care of children who have sustained trauma ${ }^{24}$.

Prior research findings indicate providers perceive individual improvement post-simulation ${ }^{14}$. Several studies report successful translation of team-oriented skills including effective communication and efficiency ${ }^{18-20}$. Participants report improvement in communication both within the immediate members of the emergency care team as well as between hospital systems ${ }^{14}$.

\section{Future Research}

Simulation-based medical education is an increasingly popular tool that facilitates experiential learning as well as the maintenance of the knowledge and skills necessary to treat high risk, rare or infrequently encountered disease processes, including traumatic injury of the pediatric population. While studies have shown that use of simulation improves provider knowledge, skills, and comfort levels, the intervals of training and longevity of evaluation provided in these studies vary significantly. Studies ranged from as few as 1 simulation followed by reassessment 6 months later to as frequent as monthly assessments for almost 2 years ${ }^{4-7,14,21-23}$. Further study to determine the optimal timeline for training and reassessment is needed. One cannot definitively say whether the knowledge and skills acquired is retained long term without the need of frequent reassessment; however, results of these studies suggest it to be so. Hunt et al completed pediatric trauma simulations in 18 EDs followed by reassessment 6 months later, resulting in marked performance improvement, indicating knowledge and skill retention over this time $\operatorname{period}^{23}$.

Additionally, while overall improvement in the simulation setting is observed, one cannot be certain that simulations are sufficiently realistic and if this truly corresponds to improvement in the clinical setting. It is not known whether a participant's performance during a simulation accurately reflects how he/she would perform during a real pediatric trauma resuscitation. While Steinemann et al, has shown that the use of simulation for teaching and assessing teamwork skills improves teamwork during actual trauma resuscitations, to our knowledge, no such comparison evaluating the translation of clinical skill has been completed. Future study needs to investigate whether simulation-based training improves the quality of pediatric trauma care provided to real patients via comparison of patient outcomes. Cost analysis of care pre and post simulation-based training would also be of benefit.

\section{Conclusions}

Simulation-based education can be used to identify and correct performance and knowledge gaps in the stabilization of children presenting to EDs as trauma patients, as well as improve provider comfort level. Simulation-based training will improve future pediatric trauma patient care and should be implemented at institutions with limited exposure to the pediatric trauma patient, especially those serving rural populations. Further study comparing patient outcomes, cost analysis, and quality improvement, prior to and after implementation of simulation-based education initiatives is warranted at all participating institutions. 


\section{References}

1. “Ten Leading Causes of Death and Injury." Centers for Disease Control and Prevention. Centers for Disease Control and Prevention, 25 Feb. 2016. Web. 02 Feb. 2017.

2. Densmore JC, Lim HJ, Oldham KT, et al. "Outcomes and delivery of care in pediatric injury.” J Pediatr Surg. 2006; 41: 92-98.

3. Guise JM, Hansen M, O'Brien K, et al. Emergency medical services responders' perceptions of the effect of stress and anxiety on patient safety in the out-of-hospital emergency care of children: a qualitative study. BMJ Open. 2017 Feb 28; 7(2): 014057. doi: 10.1136/ bmjopen-2016-014057.

4. Steinemann S, Berg B, DiTullio A, et al. Assessing teamwork in the trauma bay: introduction of a modified "NOTECHS" scale for trauma. Am J Surg. 2012; 203: 69-75.

5. Amiel I, Simon D, Merin O, Ziv A. Mobile in situ simulation as a tool for evaluation and improvement of trauma treatment in the emergency department. J Surg Educ. 2016; 73: 121-8.

6. Falcone RA Jr, Daugherty M, Schweer L, et al. Multidisciplinary pediatric trauma team training using high-fidelity trauma simulation. J Pediatr Surg. 2008; 43: 1065-71.

7. Steinemann S, Berg B, Skinner A, et al. In situ, multidisciplinary, simulation-based teamwork training improves early trauma care. J Surg Educ. 2011; 68: 472-7.

8. Petrosyan M, Guner YS, Emami CN, et al. "Disparities in the delivery of pediatric trauma care." J Trauma. 2009 Aug; 67(2 Suppl): 114-9. doi: 10.1097/TA.0b013e3181ad3251.

9. Jarman MP, Castillo RC, Carlini AR, et al. "Rural risk: Geographic disparities in trauma mortality." Surgery. 2016 Dec; 160(6): 15511559. doi: 10.1016/j.surg.2016.06.020. Epub 2016 Aug 6.

10. Curran V, Rourke L, Snow P. "A framework for enhancing continuing medical education for rural physicians: a summary of the literature." Med Teach. 2010; 32(11): 501-8.

11. Sethi D, Racioppi F, Baumgarten I, et al. "Reducing inequalities from injuries in Europe." Lancet. 2006; 368(9554): 2243-50.

12. Esposito TJ, Sanddal ND, Dean JM, et al. "Analysis of preventable pediatric trauma deaths and inappropriate trauma care in Montana." J Trauma. 1999; 47(2): 243-53.

13. Patterson MD, Blike GT, Nadkarni VM. "In situ simulation: challenges and results," in Advances in Patient Safety: New Directions and Alternative Approaches (Volume 3: Performance and Tools), Henriksen K, Battles JB, Keyes MA, et al., editors. Rockville (MD): Agency for Healthcare Research and Quality (US); 2008 Aug.

14. Bayouth L, Ashley S, Brady J, et al. "An in-situ simulation-based educational outreach project for pediatric trauma care in a rural trauma system." J Pediatr Surg. 2018 Feb; 53(2): 367-371. doi: 10.1016/j.jpedsurg.2017.10.042. Epub 2017 Oct 13.

15. Ojha R, Liu A, Rai D, et al. "Review of Simulation in Pediatrics: The Evolution of a Revolution." Front Pediatr. 2015; 3: 106. doi: 10.3389/ fped.2015.00106

16. Aggarwal R, Mytton OT, Derbrew M, et al. "Training and simulation for patient safety." Qual Saf Health Care. 2010 Aug; 19 Suppl 20: 34-43.

17. Lateef F. "Simulation-based learning: Just like the real thing." J Emerg Trauma Shock. 2010 Oct; 3(4): 348-52.

18. Lehner M, Heimberg E, Hoffmann F, et al. "Evaluation of a pilot project to introduce simulation-based team training to pediatric surgery trauma room care." Int J Ped. 2017 (2017) http://dx.doi. org/10.1155/2017/9732316.

19. Burke RV, Demeter NE, Goodhue CJ, et al. "Qualitative assessment of simulation-based training for pediatric trauma resuscitation." Surgery. 2017; 161(5): 1357-66.

20. Lin Y, Cheng A, "The role of simulation in teaching pediatric resuscitation: current perspectives," Advances in Medical Education and Practice. 2015; 6: 239-248.

21. Auerbach M, Roney L, Aysseh A, et al. "In situ pediatric trauma simulation: assessing the impact and feasibility of an interdisciplinary pediatric in situ trauma care quality improvement simulation program." Pediatr Emerg Care. 2014 Dec; 30(12): 884-91.

22. Falcone RA Jr, Daugherty M, Schweer L, et al. "Multidisciplinary pediatric trauma team training using high-fidelity trauma simulation." J Pediatr Surg. 2008 Jun; 43(6): 1065-71.

23. Hunt EA, Heine M, Hohenhaus SM, et al. Simulated pediatric trauma team management: assessment of an educational intervention. Pediatr Emerg Care. 2007 Nov; 23(11): 796-804.

24. Hunt EA, Hohenhaus SM, Luo X, et al. "Simulation of pediatric trauma stabilization in 35 North Carolina emergency departments: identification of targets for performance improvement." Pediatrics. 2006; 117(3): 641-8. 